\title{
Behaviour of linear multifractional stable motion: membership of a critical Hölder space
}

\author{
Antoine Ayache \\ Univ. Lille, CNRS, UMR 8524 - Laboratoire Paul Painlevé, \\ F-59000 Lille, France. \\ E-mail: Antoine.Ayache@math.univ-lille1.fr \\ Julien Hamonier \\ Univ. Lille, CHU Lille, EA 2694 - Santé publique : \\ épidémiologie et qualité des soins, F-59000 Lille, France. \\ E-mail: Julien.Hamonier@univ-lille2.fr
}

\begin{abstract}
The study of path behaviour of stochastic processes is a classical topic in probability theory and related areas. In this frame, a natural question one can address is: whether or not sample paths belong to a critical Hölder space? The answer to this question is negative in the case of Brownian motion and many other stochastic processes: it is well-known that despite the fact that Brownian paths satisfy, on each compact interval $I$, a Hölder condition of any order strictly less than $1 / 2$, they fail to belong to the critical Hölder space $\mathcal{C}^{1 / 2}(I)$. In this article, we show that a different phenomenon happens in the case of linear multifractional stable motion (LMSM): for any given compact interval one can find a critical Hölder space to which sample paths belong. Among other things, this result improves an upper estimate, recently derived in (Biermé, H. and Lacaux, C., 2013), on behaviour of LMSM, by showing that the logarithmic factor in it is not needed.
\end{abstract}

Mathematics Subject Classification (2010): 60G22, 60G52, 60G17.

Keywords: Linear multifractional stable motions, wavelet series representations, moduli of continuity, Hölder regularity.

\section{Introduction and statement of the main result}

Over the two last decades, there has been a growing interest in probabilistic models based on fractional and multifractional processes (see e.g. (Ayache, A. and Taqqu, M.S., 2005; Ayache, A. and Jaffard, S. and Taqqu, M.S., 2007; Ayache, A. and Shieh, N.R. and Xiao, Y., 2011; Falconer, K.J., 2002, 2003; Benassi, A. and Jaffard, S. and Roux, D., 1997; Peltier, R.F. and Lévy Véhel, J., 1995; Lacaux, C., 2004; Surgailis, D., 2008; Bianchi, S., 2005; Bianchi, S. and Pianese, A., 2008; Bianchi, S. and Pantanella, A. and Pianese, A., 2011; Lopes, R. and Ayache, A. and Makni, I 2011; Stoev, S. and Taqqu, M. S., 2004, 2005; Meerschaert, M. and Wu, D. and Xiao, Y., 2008; Bardet, J.M. and Surgailis, D., 2013; Dozzi, M. and Shevchenko, G., 2011; Hashorva, E. and Lifshits, M. and Seleznjev, O., 2015; Leonenko, N. and Ruiz-Medina, M.D. and 
2011; Stoev, S. and Taqqu, M. S., 2006; Ayache, A. and Hamonier, J., 2014; Biermé, H. and Lacaux, C., 2013; Balança, P., 2014, 2015)). Actually, they convey a convenient framework for modelling in several fields such as Internet traffic and finance.

In this article, we focus on one of the the most typical multifractional processes with heavy-tailed stable distributions: the linear multifractional stable motion (LMSM), which was first introduced in (Stoev, S. and Taqqu, M. S., 2004, 2005) and studied in (Ayache, A. and Hamonier, J., $\quad 2014$; $\quad$ Biermé, H. and Lacaux, C., $\quad 2013$; Falconer, K.J. and Le Guével, R. and Lévy Véhel, J., 2009; Falconer, K.J. and Lévy Véhel, J., 2009; Balança, P., 2014). In order to precisely define LMSM, let $\alpha \in(1 ; 2)$ be a fixed real number and $\mathrm{Z}_{\alpha}(d s)$ be an independently scattered symmetric $\alpha$-stable $(\mathcal{S} \alpha \mathcal{S})$ random measure on $\mathbb{R}$, with Lebesgue control measure (Samorodnitsky, G. and Taqqu, M. S., 1994); the underlying probability space is denoted by $(\Omega, \mathcal{F}, \mathbb{P})$. Also, let $H(\cdot)$ be an arbitrary deterministic continuous function defined on the real line with values in $(1 / \alpha ; 1)$.

Definition 1.1. The LMSM $\{Y(t): t \in \mathbb{R}\}$ with Hurst functional parameter $H(\cdot)$, is defined, for each $t \in \mathbb{R}$, as,

$$
Y(t)=X(t, H(t)),
$$

where $X:=\{X(u, v):(u, v) \in \mathbb{R} \times(1 / \alpha ; 1)\}$ is the real-valued $\mathcal{S} \alpha \mathcal{S}$ random field on the probability space $(\Omega, \mathcal{F}, \mathbb{P})$, such that, for every $(u, v) \in \mathbb{R} \times(1 / \alpha ; 1)$,

$$
X(u, v)=\int_{\mathbb{R}}\left\{(u-s)_{+}^{v-1 / \alpha}-(-s)_{+}^{v-1 / \alpha}\right\} \mathrm{Z}_{\alpha}(d s) .
$$

Recall that for all $(x, \kappa) \in \mathbb{R}^{2}$,

$$
(x)_{+}^{\kappa}=x^{\mathcal{K}} \text { if } x>0 \text { and }(x)_{+}^{\mathcal{K}}=0 \text { else. }
$$

Remark 1.1. Notice that in the special case where $H(\cdot)$ is a constant function denoted by H, LMSM reduces to linear fractional stable motion (LFSM) of Hurst parameter $H$; we mention that sample path properties of the latter process have already been studied in (Takashima, K., 1989; Maejima, M. , 1983; Kôno, N. and Maejima, M., 1991; Samorodnitsky, G. and Taqqu, M. S., 1994; Balança, P., 2014). Also notice that when $\alpha=2$ LMSM reduces to the classical multifractional Brownian motion (MBM) of functional parameter $H(\cdot)$ which was introduced in the mid 1990s independently by (Benassi, A. and Jaffard, S. and Roux, D., 1997) and by (Peltier, R.F. and Lévy Véhel, J., 1995).

In order to precisely explain the motivation behind the present article, one has to make a brief presentation of some previous results on path behaviour of LMSM which were obtained in the literature. For the sake of clarity, first, it is useful to recall the precise definition of the Hölder space $\mathcal{C}^{\gamma}(I ; \mathbb{R})$.

Definition 1.2. Let I be a compact interval of $\mathbb{R}$. For each real number $\gamma \in[0 ; 1)$, the Hölder space $\mathcal{C}^{\gamma}(I):=\mathcal{C}^{\gamma}(I ; \mathbb{R})$ is the set of the real-valued continuous functions $f$ on I satisfying:

$$
\sup _{(x, y) \in I^{2} ; x \neq y}\left\{\frac{|f(x)-f(y)|}{|x-y|^{\gamma}}\right\}<\infty .
$$

Stoev and Taqqu obtained, in their article (Stoev, S. and Taqqu, M. S., 2005), a first result about global path behaviour of LMSM on a compact interval. Namely, thanks to a strong version of the Kolmogorov continuity theorem, they showed the following result. 
Theorem 1.1. (Stoev, S. and Taqqu, M. S., 2005) Let I be a compact interval of $\mathbb{R}$. Assume that the functional parameter $H(\cdot)$ of LMSM Y satisfies:

$$
H(I) \subset(1 / \alpha ; 1) \text { and } H(\cdot) \in \mathcal{C}^{\gamma_{H}}(I) \text { for some } \gamma_{H} \in(1 / \alpha ; 1) \text {. }
$$

Then LMSM has a modification whose paths are, with probability 1 , continuous functions on I. Moreover, for all $\gamma<\min _{t \in I} H(t)-1 / \alpha$, they belong to the Hölder space $\mathcal{C}^{\gamma}(I)$

Later, thanks to a different methodology, relying on wavelet series representations of the field $X$ and the corresponding LMSM $Y$, Theorem 1.1 has been improved in the article (Ayache, A. and Hamonier, J., 2014) in the following way: under condition (A), for any $\eta>0$, one has

$$
\sup _{(t, s) \in I^{2} ; t \neq s}\left\{\frac{|Y(t)-Y(s)|}{|t-s|^{\min _{t \in I} H(t)-1 / \alpha}(1+|\log | t-s||)^{2 / \alpha+\eta}}\right\}<\infty \quad \text { a.s. }
$$

The article (Ayache, A. and Hamonier, J., 2014) has also shown that, under condition (A), the upper estimate provided by (4) on sample paths behaviour of LMSM is quasi-optimal in the following sense: for any $\eta>0$, one has

$$
\sup _{(t, s) \in I^{2} ; t \neq s}\left\{\frac{|Y(t)-Y(s)|}{|t-s|^{\min _{t \in I} H(t)-1 / \alpha}(1+|\log | t-s||)^{-\eta}}\right\}=\infty \quad \text { a.s. }
$$

Before finishing this brief presentation of previous results on path behaviour of LMSM, we mention that in their article (Biermé, H. and Lacaux, C., 2013), Biermé and Lacaux have improved, under condition (A), the upper estimate provided by (4) on sample paths behaviour of LMSM, in the following way: one has

$$
\sup _{(t, s) \in I^{2} ; t \neq s}\left\{\frac{|Y(t)-Y(s)|}{|t-s|^{\min _{t \in I} H(t)-1 / \alpha} \sqrt{1+|\log | t-s||}}\right\}<\infty \quad \text { a.s. }
$$

As a conclusion, in view of Relations (4), (5) and (6), it is natural to address the following question: assuming that condition (A) holds, do LMSM's sample paths belong to the critical Hölder space $\mathcal{C}^{\min _{t \in I} H(t)-1 / \alpha}(I)$ ?

At first sight, one is tempted to believe that the answer to such a question is negative, since this is the case for many other examples of stochastic processes. For instance, it is well-known that despite the fact that Brownian paths satisfy, on each compact interval $I$, a Hölder condition of any order strictly less than $1 / 2$, they fail to belong to the critical Hölder space $\mathcal{C}^{1 / 2}(I)$.

Yet, we will show that for LMSM the answer to the question is positive (see Corollary 1.1 given below). In fact, this will be a consequence of our main result that we are now going to state.

Theorem 1.2. We denote by I be a compact interval of $\mathbb{R}$, and we assume that the functional parameter $H(\cdot)$ of LMSM Y satisfies:

$$
\left(\mathbf{A}^{\prime}\right) \quad H(I) \subset(1 / \alpha ; 1) \text { and } H(\cdot) \in \mathcal{C}^{\gamma_{H}}(I) \text { for some } \gamma_{H} \in[0,1) \text {. }
$$

Notice that condition $\left(\mathbf{A}^{\prime}\right)$ is more general than condition $(\mathbf{A})$. Let $\Omega_{0}^{*}$ and $\Omega_{1}^{*}$ be the two events of probability 1 defined respectively in Lemma 2.2 and Proposition 2.5 given in the next section (it is clear that their intersection $\Omega_{0}^{*} \cap \Omega_{1}^{*}$ is also an event probability 1$)$. For any $\omega \in \Omega_{0}^{*} \cap \Omega_{1}^{*}$, one has

$$
\sup _{(t, s) \in I^{2} ; t \neq s}\left\{\frac{|Y(t, \omega)-Y(s, \omega)|}{|t-s|^{\gamma_{H} \wedge\left(\min _{x \in I} H(x)-1 / \alpha\right)}}\right\}<\infty .
$$


Observe that, when the functional parameter $H(\cdot)$ of LMSM $Y$ satisfies condition (A), one can derive from Relation (7) and the inequalities $\gamma_{H}>1 / \alpha>$ $\min _{x \in I} H(x)-1 / \alpha$ the following result.

Corollary 1.1. Let I be a compact interval of $\mathbb{R}$. We assume that Condition (A) holds for this interval. Then, the sample paths of LMSM Y almost surely belong to the critical Hölder space $\mathcal{C}^{\min _{t \in I} H(t)-1 / \alpha}(I)$.

Remark 1.2. In the special case of LFSM (i.e. $H(\cdot)$ is a constant function denoted by H), Corollary 1.1 has already been obtained by Takashima in (Takashima, K., 1989).

The rest of the article is organized in the following way. In Section 2, first one makes some recalls related with the wavelet series representation of the field $X$, since it provides the main tool for proving Theorem 1.2. Then one describes the strategy employed in order to get this theorem, and one states the intermediate results which are needed. The proofs of the new results among them are given in Section 3.

\section{Methodology}

Let $X:=\{X(u, v):(u, v) \in \mathbb{R} \times(1 / \alpha ; 1)\}$ be the real-valued $\mathcal{S} \alpha \mathcal{S}$ random field which has been defined in (2) and has been associated to LMSM $Y$ through (1). The main tool for proving Theorem 1.2 is the wavelet series representation of $X$ which was introduced in (Ayache, A. and Hamonier, J., 2014). First, we will make some brief recalls related with this representation of $X$. To this end, we denote by $\psi: \mathbb{R} \rightarrow \mathbb{R}$ a 3 times continuously differentiable compactly supported Daubechies mother wavelet which generates an orthonormal basis of $L^{2}(\mathbb{R})$. We mention in passing that three very classical references on the wavelet theory are the books (Daubechies, I., 1992; Meyer, Y., 1990, 1992).

The following theorem, which has been obtained in (Ayache, A. and Hamonier, J., 2014), provides the wavelet series representation of $X$.

Theorem 2.1. (Ayache, A. and Hamonier, J., 2014). Let $\Psi: \mathbb{R} \times(1 / \alpha ; 1) \rightarrow \mathbb{R}$ be the function defined, for all $(u, v) \in \mathbb{R} \times(1 / \alpha ; 1)$, as

$$
\Psi(x, v):=\int_{\mathbb{R}}(x-s)_{+}^{v-1 / \alpha} \psi(s) d s .
$$

Let $\left\{\epsilon_{j, k}:(j, k) \in \mathbb{Z}^{2}\right\}$ be the sequence of the identically distributed $\mathcal{S} \alpha \mathcal{S}$ real-valued random variables defined, for every $(j, k) \in \mathbb{Z}^{2}$, as

$$
\epsilon_{j, k}:=2^{j / \alpha} \int_{\mathbb{R}} \psi\left(2^{j} s-k\right) Z_{\alpha}(d s)
$$

Then, for any $(u, v) \in \mathbb{R} \times(1 / \alpha ; 1)$, the random variable $X(u, v)$ can be, almost surely, expressed as

$$
X(u, v)=\sum_{(j, k) \in \mathbb{Z}^{2}} 2^{-j v} \epsilon_{j, k}\left(\Psi\left(2^{j} u-k, v\right)-\Psi(-k, v)\right),
$$

where the series is on $\Omega_{0}^{*}$, the event of probability 1 introduced in Lemma 2.2 stated below, uniformly convergent in $(u, v)$, on any compact subset of $\mathbb{R} \times(1 / \alpha ; 1)$.

From now on the field $X$ is identified with its modification provided by (9). We recall that the following two lemmas, which have respectively been derived in Ayache, A. and Hamonier, J. (2014) and Ayache, A. and Roueff, F. and Xiao, Y. (2009), are the two main ingredients of the proof of Theorem 2.1. 
Lemma 2.1. (Ayache, $A$. and Hamonier, J., 2014) For all $(p, q) \in\{0,1,2,3\} \times \mathbb{Z}_{+}$, the partial derivative $\partial_{x}^{p} \partial_{v}^{q} \Psi$ exists and is a continuous function on $\mathbb{R} \times(1 / \alpha ; 1)$, with the usual convention that $\partial_{x}^{0} \partial_{v}^{0} \Psi:=\Psi$. Moreover, all of these functions ( $\Psi$ and its existing partial derivatives) are well-localized in $x$ uniformly $v \in[a ; b]$, that is one has:

$$
\forall(p, q) \in\{0,1,2,3\} \times \mathbb{Z}_{+}, \quad \sup _{(x, v) \in \mathbb{R} \times[a ; b]}\left\{(3+|x|)^{2}\left|\left(\partial_{x}^{p} \partial_{v}^{q} \Psi\right)(x, v)\right|\right\}<\infty,
$$

where $[a ; b]$ denotes an arbitrary nonempty compact subinterval of $(1 / \alpha ; 1)$.

Lemma 2.2. (Ayache, A. and Roueff, F. and Xiao, Y., 2009) There exists an event of probability 1 , denoted by $\Omega_{0}^{*}$, such that, for every fixed real number $\eta>0$, one has, for all $\omega \in \Omega_{0}^{*}$ and for each $(j, k) \in \mathbb{Z}^{2}$,

$$
\left|\epsilon_{j, k}(\omega)\right| \leq C(\omega)(3+|j|)^{1 / \alpha+\eta}(3+|k|)^{1 / \alpha+\eta},
$$

where $C$ is a positive and finite random variable only depending on $\eta$.

Remark 2.1. In view of Lemma 2.1 and of the uniform convergence property of series in (9), for each fixed $\omega \in \Omega_{0}^{*}$, the sample path $(u, v) \mapsto X(u, v, \omega)$ is a continuous function on $\mathbb{R} \times(1 / \alpha ; 1)$.

Before finishing our recalls, let us give a useful proposition which was obtained in (Ayache, A. and Hamonier, J., 2014) thanks to Lemmas 2.1 and 2.2.

Proposition 2.1. (Ayache, A. and Hamonier, J., 2014) For each fixed $\omega \in \Omega_{0}^{*}$ and real numbers $M, a, b$ satisfying $M>0$ and $1 / \alpha<a<b<1$, one has (with the convention that $0 / 0=0$ )

$$
\sup _{\left(u, v_{1}, v_{2}\right) \in[-M ; M] \times[a ; b]^{2}}\left\{\frac{\left|X\left(u, v_{1}, \omega\right)-X\left(u, v_{2}, \omega\right)\right|}{\left|v_{1}-v_{2}\right|}\right\}<\infty .
$$

Having finished our recalls related with the wavelet series representation of $X$, let us now turns to description of the strategy allowing to derive Theorem 1.2. First, we point out that this theorem is mainly a consequence of the following more general result.

Theorem 2.2. Let $\Omega_{0}^{*}$ be the event of probability 1 which has already been introduced in Lemma 2.2. Let $\Omega_{1}^{*}$ be the event of probability 1 which will be introduced in Proposition 2.5 given below. For all fixed $\omega \in \Omega_{0}^{*} \cap \Omega_{1}^{*}$ and real numbers $M, a, b$ satisfying $M>0$ and $1 / \alpha<a<b<1$, one has (with the convention that $0 / 0=0$ )

$$
\sup _{\left(u_{1}, u_{2}, v_{1}, v_{2}\right) \in[-M ; M]^{2} \times[a ; b]^{2}}\left\{\frac{\left|X\left(u_{1}, v_{1}, \omega\right)-X\left(u_{2}, v_{2}, \omega\right)\right|}{\left|u_{1}-u_{2}\right|^{v_{1} \vee v_{2}-1 / \alpha}+\left|v_{1}-v_{2}\right|}\right\}<+\infty,
$$

where $v_{1} \vee v_{2}:=\max \left(v_{1}, v_{2}\right)$.

We mention that, even though it is more general than Theorem 1.2, we have prefered to state Theorem 2.2 in the present section, and not in the introductory section, since it is a bit technical result. Let us now explain how Theorem 2.2 allows to obtain Theorem 1.2.

Proof of Theorem 1.2. There is no restriction to assume that the compact interval $I$ is of the form $I=[-M ; M]$, where $M$ is some positive real number. Let us then consider an arbitrary $\omega \in \Omega_{0}^{*} \cap \Omega_{1}^{*}$ and an arbitrary couple $(t, s) \in[-M ; M]^{2}$, such 
that $t \neq s$. Using Relation (1), Theorem 2.2, condition $\left(\mathbf{A}^{\prime}\right)$, and Definition 1.2, one gets that

$$
\begin{aligned}
|Y(t, \omega)-Y(s, \omega)| & =|X(t, H(t), \omega)-X(s, H(s), \omega)| \\
& \leq C(\omega)\left(|t-s|^{H(t) \vee H(s)-1 / \alpha}+|H(t)-H(s)|\right) \\
& \leq C(\omega)|t-s|^{H(t) \vee H(s)-1 / \alpha}+C^{\prime}(\omega)|t-s|^{\gamma_{H}} \\
& \leq C^{\prime \prime}(\omega)|t-s|^{\min _{x \in I} H(x)-1 / \alpha}+C^{\prime}(\omega)|t-s|^{\gamma_{H}} \\
& \leq C^{\prime \prime \prime}(\omega)|t-s|^{\gamma_{H} \wedge\left(\min _{x \in I} H(x)-1 / \alpha\right)},
\end{aligned}
$$

where $C, \ldots, C^{\prime \prime \prime}$ are positive and finite random variables not depending on $(t, s)$.

Theorem 2.2 can be derived from Proposition 2.1 and the following proposition.

Proposition 2.2. For all fixed $\omega \in \Omega_{0}^{*} \cap \Omega_{1}^{*}$ and real numbers $M, a, b$ satisfying $M>0$ and $1 / \alpha<a<b<1$, one has (with the convention that $0 / 0=0$ )

$$
\sup _{\left(u_{1}, u_{2}, v\right) \in[-M ; M]^{2} \times[a ; b]}\left\{\frac{\left|X\left(u_{1}, v, \omega\right)-X\left(u_{2}, v, \omega\right)\right|}{\left|u_{1}-u_{2}\right|^{v-1 / \alpha}}\right\}<\infty .
$$

Proof of Theorem 2.2. For all $\omega \in \Omega_{0}^{*} \cap \Omega_{1}^{*}$ and for each $\left(u_{1}, u_{2}, v_{1}, v_{2}\right) \in[-M ; M]^{2} \times$ $[a ; b]^{2}$, one sets

$$
f\left(u_{1}, u_{2}, v_{1}, v_{2}, \omega\right):=\frac{\left|X\left(u_{1}, v_{1}, \omega\right)-X\left(u_{2}, v_{2}, \omega\right)\right|}{\left|u_{1}-u_{2}\right|^{v_{1} \vee v_{2}-1 / \alpha}+\left|v_{1}-v_{2}\right|},
$$

with the convention that $0 / 0=0$. Next setting $v_{1} \wedge v_{2}:=\min \left(v_{1}, v_{2}\right)$, and using the fact that

$$
f\left(u_{1}, u_{2}, v_{1}, v_{2}, \omega\right)=f\left(u_{2}, u_{1}, v_{2}, v_{1}, \omega\right),
$$

it follows that

$$
\begin{array}{ll}
\sup _{\left(u_{1}, u_{2}, v_{1}, v_{2}\right) \in[-M ; M]^{2} \times[a ; b]^{2}} & \left\{\frac{\left|X\left(u_{1}, v_{1}, \omega\right)-X\left(u_{2}, v_{2}, \omega\right)\right|}{\left|u_{1}-u_{2}\right|^{v_{1} \vee v_{2}-1 / \alpha}+\left|v_{1}-v_{2}\right|}\right\} \\
= & \sup _{\left(u_{1}, u_{2}, v_{1}, v_{2}\right) \in[-M ; M]^{2} \times[a ; b]^{2}}\left\{\frac{\left|X\left(u_{1}, v_{1} \vee v_{2}, \omega\right)-X\left(u_{2}, v_{1} \wedge v_{2}, \omega\right)\right|}{\left|u_{1}-u_{2}\right|^{v_{1} \vee v_{2}-1 / \alpha}+\left|v_{1}-v_{2}\right|}\right\} .
\end{array}
$$

Moreover, using the triangle inequality, and the inequality, for all $\left(u_{1}, u_{2}, v_{1}, v_{2}\right) \in$ $[-M ; M]^{2} \times[a ; b]^{2}$,

$$
\max \left\{\left|u_{1}-u_{2}\right|^{v_{1} \vee v_{2}-1 / \alpha},\left|v_{1}-v_{2}\right|\right\} \leq\left|u_{1}-u_{2}\right|^{v_{1} \vee v_{2}-1 / \alpha}+\left|v_{1}-v_{2}\right|,
$$


one gets that

$$
\begin{aligned}
& \operatorname{cup}_{\left(u_{1}, u_{2}, v_{1}, v_{2}\right) \in[-M ; M]^{2} \times[a ; b]^{2}}\left\{\frac{\left|X\left(u_{1}, v_{1} \vee v_{2}, \omega\right)-X\left(u_{2}, v_{1} \wedge v_{2}, \omega\right)\right|}{\left|u_{1}-u_{2}\right|^{v_{1} \vee v_{2}-1 / \alpha}+\left|v_{1}-v_{2}\right|}\right\} \\
& \leq \sup _{\left(u_{1}, u_{2}, v_{1}, v_{2}\right) \in[-M ; M]^{2} \times[a ; b]^{2}}\left\{\frac{\left|X\left(u_{1}, v_{1} \vee v_{2}, \omega\right)-X\left(u_{2}, v_{1} \vee v_{2}, \omega\right)\right|}{\left|u_{1}-u_{2}\right|^{v_{1} \vee v_{2}-1 / \alpha}+\left|v_{1}-v_{2}\right|}\right\} \\
& +\sup _{\left(u_{1}, u_{2}, v_{1}, v_{2}\right) \in[-M ; M]^{2} \times[a ; b]^{2}}\left\{\frac{\left|X\left(u_{2}, v_{1} \vee v_{2}, \omega\right)-X\left(u_{2}, v_{1} \wedge v_{2}, \omega\right)\right|}{\left|u_{1}-u_{2}\right|^{v_{1} \vee v_{2}-1 / \alpha}+\left|v_{1}-v_{2}\right|}\right\} \\
& \leq \sup _{\left(u_{1}, u_{2}, v\right) \in[-M ; M]^{2} \times[a ; b]}\left\{\frac{\left|X\left(u_{1}, v, \omega\right)-X\left(u_{2}, v, \omega\right)\right|}{\left.\left|u_{1}-u_{2}\right|^{v-1 / \alpha}\right\}}\right. \\
& +\sup _{\left(u, v_{1}, v_{2}\right) \in[-M ; M] \times[a ; b]^{2}}\left\{\frac{\left|X\left(u, v_{1}, \omega\right)-X\left(u, v_{2}, \omega\right)\right|}{\left|v_{1}-v_{2}\right|}\right\} .
\end{aligned}
$$

Finally, combining (14) and (15) with Propositions 2.1 and 2.2 together, one obtains (13).

For proving Proposition 2.2 one splits the field $X$ in two parts denoted by $\dot{X}:=\{\dot{X}(u, v):(u, v) \in \mathbb{R} \times(1 / \alpha ; 1)\}$ and $\ddot{X}:=\{\ddot{X}(u, v):(u, v) \in \mathbb{R} \times(1 / \alpha ; 1)\} . \dot{X}$ is called the low frequency part, and $\ddot{X}$ the high frequency part. These two $\mathcal{S} \alpha \mathcal{S}$ random fields are defined, by setting, for all $(u, v) \in \mathbb{R} \times(1 / \alpha ; 1)$,

$$
\begin{aligned}
\dot{X}(u, v) & =\sum_{j=-\infty}^{-1} \sum_{k \in \mathbb{Z}} 2^{-j v} \epsilon_{j, k}\left(\Psi\left(2^{j} u-k, v\right)-\Psi(-k, v)\right) \\
& =\sum_{j=1}^{+\infty} \sum_{k \in \mathbb{Z}} 2^{j v} \epsilon_{-j, k}\left(\Psi\left(2^{-j} u-k, v\right)-\Psi(-k, v)\right),
\end{aligned}
$$

and

$$
\ddot{X}(u, v)=\sum_{j=0}^{+\infty} \sum_{k \in \mathbb{Z}} 2^{-j v} \epsilon_{j, k}\left(\Psi\left(2^{j} u-k, v\right)-\Psi(-k, v)\right) .
$$

Remark 2.2. One can show that, on the event $\Omega_{0}^{*}$, the two series defining $\dot{X}$ and $\ddot{X}$ share exactly the same uniform convergence property with respect to $(u, v)$ as the series in (9). Therefore, in view of Lemma 2.1, for each fixed $\omega \in \Omega_{0}^{*}$, the sample paths $(u, v) \mapsto \dot{X}(u, v, \omega)$ and $(u, v) \mapsto \ddot{X}(u, v, \omega)$ are continuous functions on $\mathbb{R} \times(1 / \alpha ; 1)$.

Proof of Proposition 2.2. One clearly has that

$$
X(u, v, \omega)=\dot{X}(u, v, \omega)+\ddot{X}(u, v, \omega),
$$

for all $(u, v) \in \mathbb{R} \times(1 / \alpha ; 1)$ and $\omega \in \Omega_{0}^{*}$. Therefore Proposition 2.2 is a straightforward consequence of the following two propositions.

Proposition 2.3. For each fixed $\omega \in \Omega_{0}^{*}$, the sample path $(u, v) \mapsto \dot{X}(u, v, \omega)$ is a 3 times continuously differentiable function on $\mathbb{R} \times(1 / \alpha ; 1)$. Therefore, it is a Lipschitz function on every compact rectangle $[-M ; M] \times[a ; b] \subset \mathbb{R} \times(1 / \alpha ; 1)$; in other words, one has that (with the convention that $0 / 0=0$ )

$$
\sup _{\left(u_{1}, u_{2}, v_{1}, v_{2}\right) \in[-M ; M]^{2} \times[a ; b]^{2}}\left\{\frac{\left|\dot{X}\left(u_{1}, v_{1}, \omega\right)-\dot{X}\left(u_{2}, v_{2}, \omega\right)\right|}{\left|u_{1}-u_{2}\right|+\left|v_{1}-v_{2}\right|}\right\}<\infty,
$$


and consequently that

$$
\sup _{\left(u_{1}, u_{2}, v\right) \in[-M ; M]^{2} \times[a ; b]}\left\{\frac{\left|\dot{X}\left(u_{1}, v, \omega\right)-\dot{X}\left(u_{2}, v, \omega\right)\right|}{\left|u_{1}-u_{2}\right|}\right\}<\infty .
$$

Proposition 2.4. For all fixed $\omega \in \Omega_{0}^{*} \cap \Omega_{1}^{*}$ and any real numbers $M, a, b$ satisfying $M>0$ and $1 / \alpha<a<b<1$, one has (with the convention that $0 / 0=0$ )

$$
\sup _{\left(u_{1}, u_{2}, v\right) \in[-M ; M]^{2} \times[a ; b]}\left\{\frac{\left|\ddot{X}\left(u_{1}, v, \omega\right)-\ddot{X}\left(u_{2}, v, \omega\right)\right|}{\left|u_{1}-u_{2}\right|^{v-1 / \alpha}}\right\}<\infty .
$$

These two propositions are proved in Section 3.

We mention that the strategy of the proof of Proposition 2.3 is to show that on the event $\Omega_{0}^{*}$, the uniform convergence property with respect to $(u, v)$ of the series in (16) is preserved when the partial derivative operator $\partial_{x}^{p} \partial_{v}^{q}$, with $(p, q) \in$ $\{0,1,2,3\} \times \mathbb{Z}^{+}$, is applied to each term of the series.

Let us emphasize that the keystone of the proof of the crucial Proposition 2.4 consists in sharpening the estimate of $\epsilon_{j, k}$ provided by (11), when $j \geq 0$ and $|k| 2^{-j}$ is bounded by an arbitrary finite constant not depending on $(j, k)$. More precisely the keystone consists in the following proposition.

Proposition 2.5. There exists an event of probability 1 , denoted by $\Omega_{1}^{*}$, such that, for every fixed positive real number $l$, one has, for all $\omega \in \Omega_{1}^{*}$ and for each $(j, k) \in \mathbb{Z}^{+} \times \mathbb{Z}$ satisfying $|k| 2^{-j} \leq l$,

$$
\left|\epsilon_{j, k}(\omega)\right| \leq C^{\prime}(\omega) 2^{j / \alpha},
$$

where $C^{\prime}$ is positive and finite random variable.

The proof of Proposition 2.5 will be given in Section 3. We mention that it relies on the following lemma which can be viewed as a integration by parts formula between a twice differentiable compactly supported function and a Lévy $\mathcal{S} \alpha \mathcal{S}$ process. The proof of this lemma will also be given in Section 3.

Lemma 2.3. Let $f: \mathbb{R} \rightarrow \mathbb{R}$ be a twice times continuously differentiable compactly supported function, and let $\mathrm{Z}_{\alpha}(\cdot)$ be a $\mathcal{S} \alpha \mathcal{S}$ random measure with a parameter $\alpha$ belonging to $(1 ; 2)$. Then, one has, almost surely

$$
\int_{\mathbb{R}} f(s) Z_{\alpha}(d s)=-\int_{\mathbb{R}} f^{\prime}(s) Z_{\alpha}(s) d s,
$$

where $\mathrm{Z}_{\alpha}(s):=\mathrm{Z}_{\alpha}([0 ; s])$ if $s \geq 0$ and $\mathrm{Z}_{\alpha}(s):=-\mathrm{Z}_{\alpha}([\mathrm{s} ; 0])$ otherwise. Notice that the stochastic process $\left\{Z_{\alpha}(s): s \in \mathbb{R}\right\}$ defined in this way is a Lévy $\mathcal{S} \alpha \mathcal{S}$ process which we always identify with its càdlàg modification.

\section{Proofs of intermediate results}

The proofs of the intermediate results are given in the following natural order: firstly that of Lemma 2.3, secondly that of Proposition 2.5, thirdly that of Proposition 2.4, and finally that of Proposition 2.3.

Proof of Lemma 2.3. Let $a<b$ be two fixed real numbers such that

$$
\operatorname{supp}(f) \subseteq[a ; b]
$$


For any fixed integer $j \geq 0$, one denotes by $\left(y_{j, k}\right)_{0 \leq k \leq 2^{j}}$ the partition of the interval $[a ; b]$ such that

$$
y_{j, k}:=a+k 2^{-j}(b-a), \quad \text { for all } k \in\left\{0, \ldots, 2^{j}\right\} .
$$

Moreover, one denotes by $\theta_{j}$ the step function defined as

$$
\theta_{j}(s):=\sum_{k=1}^{2^{j}} f\left(y_{j, k}\right) \mathbb{1}_{\left(y_{j, k-1} ; y_{j, k}\right]}(s), \quad \text { for all } s \in \mathbb{R} .
$$

Let now show that

$$
\lim _{j \rightarrow+\infty}\left\|f-\theta_{j}\right\|_{L^{\alpha}(\mathbb{R})}=0 .
$$

Using the definition of the $\mathrm{L}^{\alpha}(\mathbb{R})$-norm, (20), (22), the mean value theorem and (21), one obtains that

$$
\begin{aligned}
\left\|f-\theta_{j}\right\|_{L^{\alpha}(\mathbb{R})}^{\alpha} & :=\int_{\mathbb{R}}\left|f(s)-\theta_{j}(s)\right|^{\alpha} d s=\int_{\mathbb{R}}\left|\sum_{k=1}^{2^{j}}\left(f(s)-f\left(y_{j, k}\right)\right) \mathbb{1}_{\left(y_{j, k-1} ; y_{j, k}\right]}(s)\right|^{\alpha} d s \\
& =\sum_{k=1}^{2^{j}} \int_{\mathbb{R}}\left|f(s)-f\left(y_{j, k}\right)\right|^{\alpha} \mathbb{1}_{\left(y_{j, k-1} ; y_{j, k}\right]}(s) d s \\
& \leq\left(\sup _{s \in[a ; b]}\left|f^{\prime}(s)\right|^{\alpha}\right) \sum_{k=1}^{2^{j}} \int_{\mathbb{R}}\left(y_{k}-s\right)^{\alpha} \mathbb{1}_{\left(y_{j, k-1} ; y_{j, k}\right]}(s) d s \\
& =\left(\sup _{s \in[a ; b]}\left|f^{\prime}(s)\right|^{\alpha}\right)(\alpha+1)^{-1}(b-a)^{\alpha+1} \sum_{k=1}^{2^{j}} 2^{-j(\alpha+1)} \\
& =\left(\sup _{s \in[a ; b]}\left|f^{\prime}(s)\right|^{\alpha}\right)(\alpha+1)^{-1}(b-a)^{\alpha+1} 2^{-j \alpha},
\end{aligned}
$$

which shows that (23) is satisfied. Then, one can deduce from (23) and from a classical property of an $\alpha$-stable stochastic integral, with $\alpha>1$, (see (Samorodnitsky, G. and Taqqu, M. S., 1994)) that

$$
\lim _{j \rightarrow+\infty} \mathbb{E}\left|\int_{\mathbb{R}} f(s) Z_{\alpha}(d s)-\int_{\mathbb{R}} \theta_{j}(s) Z_{\alpha}(d s)\right|=0 .
$$

On the other hand, using (22), standard computations and elementary properties of a stable stochastic integral, one gets that

$$
\begin{aligned}
& \int_{\mathbb{R}} \theta_{j}(s) \mathrm{Z}_{\alpha}(d s)=\sum_{k=1}^{2^{j}} f\left(y_{j, k}\right)\left(\mathrm{Z}_{\alpha}\left(y_{j, k}\right)-\mathrm{Z}_{\alpha}\left(y_{j, k-1}\right)\right) \\
& =f\left(y_{j, 2^{j}}\right) \mathrm{Z}_{\alpha}\left(y_{j, 2^{j}}\right)-f\left(y_{j, 1}\right) \mathrm{Z}_{\alpha}\left(y_{j, 0}\right)-\sum_{k=1}^{2^{j}-1}\left(f\left(y_{j, k+1}\right)-f\left(y_{j, k}\right)\right) \mathrm{Z}_{\alpha}\left(y_{j, k}\right) .
\end{aligned}
$$

Notice that the inclusion (20) implies that $f\left(y_{j, 2^{j}}\right) Z_{\alpha}\left(y_{j, 2^{j}}\right)=f(b) Z_{\alpha}(b)=0$ and that $\mathbb{E}\left|f\left(y_{j, 1}\right) \mathrm{Z}_{\alpha}\left(y_{j, 0}\right)\right|=\mathbb{E}\left|f\left(a+2^{-j}(b-a)\right) \mathrm{Z}_{\alpha}(a)\right| \rightarrow 0$, when $j \rightarrow+\infty$. Also notice 
that it follows from Taylor formula that

$$
\begin{aligned}
& \sum_{k=1}^{2^{j}-1}\left(f\left(y_{j, k+1}\right)-f\left(y_{j, k}\right)\right) \mathrm{Z}_{\alpha}\left(y_{j, k}\right) \\
& =\sum_{k=1}^{2^{j}-1}\left(y_{j, k+1}-y_{j, k}\right) f^{\prime}\left(y_{j, k}\right) \mathrm{Z}_{\alpha}\left(y_{j, k}\right) \\
& \quad+\sum_{k=1}^{2^{j}-1}\left(y_{j, k+1}-y_{j, k}\right)^{2} \mathrm{Z}_{\alpha}\left(y_{j, k}\right) \int_{0}^{1}(1-s) f^{\prime \prime}\left(y_{j, k}+s\left(y_{j, k+1}-y_{j, k}\right)\right) d s .
\end{aligned}
$$

Let us now show that the absolute first moment of the last sum converges to 0 when $j \rightarrow+\infty$. Using the triangle inequality, the inequalities $\sup _{x \in[a ; b]}\left|f^{\prime \prime}(x)\right|<\infty$ and $\sup _{x \in[a ; b]} \mathbb{E}\left|Z_{\alpha}(x)\right|<\infty$, and the equality (21), one obtains that

$$
\begin{aligned}
& \mathbb{E}\left|\sum_{k=1}^{2^{j}-1}\left(y_{j, k+1}-y_{j, k}\right)^{2} Z_{\alpha}\left(y_{j, k}\right) \int_{0}^{1}(1-s) f^{\prime \prime}\left(y_{j, k}+s\left(y_{j, k+1}-y_{j, k}\right)\right) d s\right| \\
& \leq\left(\sup _{x \in[a ; b]}\left|f^{\prime \prime}(x)\right|\right)\left(\sup _{x \in[a ; b]} \mathbb{E}\left|Z_{\alpha}(x)\right|\right) \sum_{k=1}^{2^{j}-1}\left(y_{j, k+1}-y_{j, k}\right)^{2} \\
& \leq(b-a)^{2}\left(\sup _{x \in[a ; b]}\left|f^{\prime \prime}(x)\right|\right)\left(\sup _{x \in[a ; b]} \mathbb{E}\left|Z_{\alpha}(x)\right|\right) 2^{-j} \rightarrow 0, \text { when } j \rightarrow+\infty .
\end{aligned}
$$

In order to complete our proof, it remains to show that

$$
\lim _{j \rightarrow+\infty} \mathbb{E}\left|\sum_{k=1}^{2^{j}-1}\left(y_{j, k+1}-y_{j, k}\right) f^{\prime}\left(y_{j, k}\right) Z_{\alpha}\left(y_{j, k}\right)-\int_{a}^{b} f^{\prime}(s) Z_{\alpha}(s) d s\right|=0 .
$$

It follows from the equality $f^{\prime}\left(y_{j, 0}\right)=f^{\prime}(a)=0$ and from elementary properties of a stable stochastic integral that

$$
\sum_{k=1}^{2^{j}-1}\left(y_{j, k+1}-y_{j, k}\right) f^{\prime}\left(y_{j, k}\right) \mathrm{Z}_{\alpha}\left(y_{j, k}\right)=\int_{a}^{b}\left(\sum_{k=0}^{2^{j}-1} f^{\prime}\left(y_{j, k}\right) \mathrm{Z}_{\alpha}\left(y_{j, k}\right) \mathbb{1}_{\left(y_{j, k} ; y_{j, k+1}\right]}(s)\right) d s .
$$

Thus, using the Fubini-Tonelli theorem and (21), it turns out that for deriving (24) it is enough to show that

$$
\lim _{j \rightarrow+\infty} 2^{-j} \sum_{k=0}^{2^{j}-1} \sup _{s \in\left[y_{j, k} ; y_{j, k+1}\right]}\left\{\mathbb{E}\left|f^{\prime}\left(y_{j, k}\right) Z_{\alpha}\left(y_{j, k}\right)-f^{\prime}(s) Z_{\alpha}(s)\right|\right\}=0 .
$$

It results from properties of $f^{\prime}$ and $Z_{\alpha}$ that there exists a finite constant $c$ such that, for all $(x, y) \in[a ; b]^{2}$, one has

$$
\left|f^{\prime}(x)-f^{\prime}(y)\right| \leq c|x-y| \text { and } \mathbb{E}\left|Z_{\alpha}(x)-Z_{\alpha}(y)\right| \leq c|x-y|^{1 / \alpha} .
$$

Finally, using these two inequalities, the equality (21), and standard computations one can get (25).

Proof of Proposition 2.5. First observe that, it follows from (8), Lemma 2.3 and the change of variable $u=2^{j} s-k$, that one has, almost surely,

$$
\epsilon_{j, k}:=2^{j / \alpha} \int_{\mathbb{R}} \psi\left(2^{j} s-k\right) Z_{\alpha}(d s)=-2^{j / \alpha} \int_{\mathbb{R}} \psi^{\prime}(u) Z_{\alpha}\left((u+k) 2^{-j}\right) d u .
$$


Next, let $R$ be a fixed positive real number such that $\operatorname{supp}(\psi) \subseteq[-R ; R]$. Then using (26) and the assumption that $|k| 2^{-j} \leq l$, one gets, almost surely, that

$$
\left|\epsilon_{j, k}\right| \leq C^{\prime} 2^{j / \alpha}
$$

where $C^{\prime}$ is the positive and finite random variable, not depending on $(j, k)$, defined, almost surely, as

$$
C^{\prime}:=\left(\sup _{|x| \leq R+l}\left|Z_{\alpha}(x)\right|\right) \times\left(\int_{-R}^{R}\left|\psi^{\prime}(u)\right| d u\right) .
$$

Notice that the almost sure finiteness of $C^{\prime}$ is mainly a consequence of the fact that the sample paths of the Lévy $\mathcal{S} \alpha \mathcal{S}$ process $\left\{Z_{\alpha}(s): s \in \mathbb{R}\right\}$ are, with probability 1 , càdlàg functions on $\mathbb{R}$.

Proof of Proposition 2.4. First observe that in order to show that (18) is satisfied, it is enough to prove that

$$
\begin{aligned}
\sup \left\{\frac{\left|\ddot{X}\left(u_{1}, v, \omega\right)-\ddot{X}\left(u_{2}, v, \omega\right)\right|}{\left|u_{1}-u_{2}\right|^{v-1 / \alpha}}:\left(u_{1}, u_{2}, v\right) \in[-M ; M]^{2} \times[a ; b]\right. \\
\text { and } \left.\left|u_{1}-u_{2}\right|>1\right\}<\infty
\end{aligned}
$$

and

$$
\begin{aligned}
\sup \left\{\frac{\left|\ddot{X}\left(u_{1}, v, \omega\right)-\ddot{X}\left(u_{2}, v, \omega\right)\right|}{\left|u_{1}-u_{2}\right|^{v-1 / \alpha}}:\left(u_{1}, u_{2}, v\right) \in[-M ; M]^{2} \times[a ; b]\right. \\
\text { and } \left.0<\left|u_{1}-u_{2}\right| \leq 1\right\}<\infty
\end{aligned}
$$

Notice that Remark 2.2 in Section 2 easily implies that (27) is satisfied. So from now on, we focus on (28).

Let $\left(u_{1}, u_{2}, v\right) \in[-M ; M]^{2} \times[a ; b]$ be arbitrary but such that $0<\left|u_{1}-u_{2}\right| \leq 1$. In view of Relation (10), there exists a constant $c>0$, which does not depend on $u_{1}, u_{2}$ and $v$, such that, for all $(j, k) \in \mathbb{Z}^{+} \times \mathbb{Z}$, one has

$$
\left|\Psi\left(2^{j} u_{1}-k, v\right)-\Psi\left(2^{j} u_{2}-k, v\right)\right| \leq c_{1}\left(\left(3+\left|2^{j} u_{1}-k\right|\right)^{-2}+\left(3+\left|2^{j} u_{2}-k\right|\right)^{-2}\right) .
$$

Moreover,

$$
\left|\Psi\left(2^{j} u_{1}-k, v\right)-\Psi\left(2^{j} u_{2}-k, v\right)\right|
$$

can be bounded more sharply when the condition

$$
2^{j}\left|u_{1}-u_{2}\right| \leq 1
$$

holds. More precisely, using the mean value theorem and (10), one has

$$
\begin{aligned}
& \left|\Psi\left(2^{j} u_{1}-k, v\right)-\Psi\left(2^{j} u_{2}-k, v\right)\right| \\
& \leq 2^{j}\left|u_{1}-u_{2}\right| \sup _{(u, v) \in\left[u_{1} \wedge u_{2}, u_{1} \vee u_{2}\right] \times[a, b]}\left|\left(\partial_{x} \Psi\right)\left(2^{j} u-k, v\right)\right| \\
& \leq c 2^{j}\left|u_{1}-u_{2}\right| \sup _{u \in\left[u_{1} \wedge u_{2}, u_{1} \vee u_{2}\right]}\left(3+\left|2^{j} u-k\right|\right)^{-2} \leq c 2^{j}\left|u_{1}-u_{2}\right|\left(2+\left|2^{j} u_{1}-k\right|\right)^{-2} .
\end{aligned}
$$

Next, one denotes by $j_{0}$ the greater integer such that

$$
2^{-1}<2^{j_{0}}\left|u_{1}-u_{2}\right| \leq 1
$$


Observe that one necessarily has that $j_{0} \geq 0$ since $\left|u_{1}-u_{2}\right| \leq 1$. By combining together (29), (31) and (32) together, one gets

$$
\begin{aligned}
& \sum_{j \geq 0} 2^{-j v} \sum_{k \in \mathbb{Z}}\left|\epsilon_{j, k}\right|\left|\Psi\left(2^{j} u_{1}-k, v\right)-\Psi\left(2^{j} u_{2}-k, v\right)\right| \\
& \leq c\left(A_{j_{0}}\left(u_{1}, v\right) 2^{-j_{0}}+B_{j_{0}}\left(u_{1}, u_{2}, v\right)\right),
\end{aligned}
$$

where

$$
A_{j_{0}}\left(u_{1}, v\right)=\sum_{j=0}^{j_{0}} 2^{j(1-v)} \sum_{k \in \mathbb{Z}}\left|\epsilon_{j, k}\right|\left(2+\left|2^{j} u_{1}-k\right|\right)^{-2}
$$

and

$$
B_{j_{0}}\left(u_{1}, u_{2}, v\right)=\sum_{j=j_{0}+1}^{+\infty} 2^{-j v} \sum_{k \in \mathbb{Z}}\left|\epsilon_{j, k}\right|\left(\left(3+\left|2^{j} u_{1}-k\right|\right)^{-2}+\left(3+\left|2^{j} u_{2}-k\right|\right)^{-2}\right) .
$$

In order to provide appropriate upper bounds for $A_{j_{0}}\left(u_{1}, v\right) 2^{-j_{0}}$ and $B_{j_{0}}\left(u_{1}, u_{2}, v\right)$, one needs to introduce, for all fixed integer $j \geq 0$, the two sets of indices $k, D_{j}^{1}$ and $D_{j}^{2}$, defined as

$$
D_{j}^{1}:=\left\{k \in \mathbb{Z}:|k| 2^{-j} \leq 2(M+1)\right\} \text { and } D_{j}^{2}:=\left\{k \in \mathbb{Z}:|k| 2^{-j}>2(M+1)\right\} .
$$

Notice that $\mathbb{Z}=D_{j}^{1} \cup D_{j}^{2}$ and $D_{j}^{1} \cap D_{j}^{2}=\emptyset$.

Let us first provide an appropriate upper bound for $A_{j_{0}}\left(u_{1}, v\right) 2^{-j_{0}}$. On one hand Relation (11) and standard computations give us

$$
\begin{aligned}
& \sum_{j=0}^{j_{0}} 2^{j(1-v)} \sum_{k \in D_{j}^{2}}\left|\epsilon_{j, k}\right|\left(2+\left|2^{j} u_{1}-k\right|\right)^{-2} \\
& \leq C \sum_{j=0}^{j_{0}} 2^{j(1-v)}(3+j)^{1 / \alpha+\eta} \sum_{k \in D_{j}^{2}} \frac{(3+|k|)^{1 / \alpha+\eta}}{\left(2+\left|2^{j} u_{1}-k\right|\right)^{2}} \\
& \leq C \sum_{j=0}^{j_{0}} 2^{j(1-v)}(3+j)^{1 / \alpha+\eta} \sum_{k \in D_{j}^{2}}(1+|k|)^{-(2-1 / \alpha-\eta)} \\
& \leq C \sum_{j=0}^{j_{0}} 2^{j(1-v)}(3+j)^{1 / \alpha+\eta} 2^{-j(1-1 / \alpha-\eta)}=C \sum_{j=0}^{j_{0}} 2^{-j(v-1 / \alpha-\eta)}(3+j)^{1 / \alpha+\eta} \\
& \leq C \sum_{j=0}^{j_{0}} 2^{-j(a-1 / \alpha-\eta)}(3+j)^{1 / \alpha+\eta} \leq C \sum_{j=0}^{+\infty} 2^{-j(a-1 / \alpha-\eta)}(3+j)^{1 / \alpha+\eta}=C_{1}<\infty ;
\end{aligned}
$$

notice that in the previous inequalities, and in the rest of this proof, $C$ denotes the same positive and finite random variable as in (11). On the other hand, using Proposition 2.5, one has

$$
\begin{aligned}
& \sum_{j=0}^{j_{0}} 2^{j(1-v)} \sum_{k \in D_{j}^{1}}\left|\epsilon_{j, k}\right|\left(2+\left|2^{j} u_{1}-k\right|\right)^{-2} \leq C^{\prime} \sum_{j=0}^{j_{0}} 2^{j(1-v+1 / \alpha)} \sum_{k \in D_{j}^{1}}\left(2+\left|2^{j} u_{1}-k\right|\right)^{-2} \\
& \leq C^{\prime} \sup _{x \in[0,1]}\left(\sum_{k \in \mathbb{Z}}(1+|x-k|)^{-2}\right) \sum_{j=0}^{j_{0}} 2^{j(1-v+1 / \alpha)} \leq C_{2} 2^{j_{0}(1-v+1 / \alpha)} .
\end{aligned}
$$


notice that in the previous inequalities $C^{\prime}$, and in the rest of this proof, denotes the same positive and finite random variable as in (19). Next, it follows from (34), (37), (38) and (32) that

$$
\begin{aligned}
& A_{j_{0}}\left(u_{1}, v\right) 2^{-j_{0}}=\left(\sum_{j=0}^{j_{0}} 2^{j(1-v)} \sum_{k \in D_{j}^{1}}\left|\epsilon_{j, k}\right|\left(2+\left|2^{j} u_{1}-k\right|\right)^{-2}\right. \\
&\left.+\sum_{j=0}^{j_{0}} 2^{j(1-v)} \sum_{k \in D_{j}^{2}}\left|\epsilon_{j, k}\right|\left(2+\left|2^{j} u_{1}-k\right|\right)^{-2}\right) 2^{-j_{0}} \\
& \leq\left(C_{1}+C_{2} 2^{j_{0}(1-v+1 / \alpha)}\right) 2^{-j_{0}} \leq C_{3}\left|u_{1}-u_{2}\right|^{v-1 / \alpha} .
\end{aligned}
$$

Let us now provide an appropriate upper bound for $B_{j_{0}}\left(u_{1}, u_{2}, v\right)$. On one hand, using Relation (11) and standard computations one gets

$$
\begin{aligned}
& \sum_{j=j_{0}+1}^{+\infty} 2^{-j v} \sum_{k \in D_{j}^{2}}\left|\epsilon_{j, k}\right|\left(\left(3+\left|2^{j} u_{1}-k\right|\right)^{-2}+\left(3+\left|2^{j} u_{2}-k\right|\right)^{-2}\right) \\
& \leq C \sum_{j=j_{0}+1}^{+\infty} 2^{-j v}(3+|j|)^{1 / \alpha+\eta} \sum_{k \in D_{j}^{2}}\left(\frac{(3+|k|)^{1 / \alpha+\eta}}{\left(3+\left|2^{j} u_{1}-k\right|\right)^{2}}+\frac{(3+|k|)^{1 / \alpha+\eta}}{\left(3+\left|2^{j} u_{2}-k\right|\right)^{2}}\right) \\
& \leq C \sum_{j=j_{0}+1}^{+\infty} 2^{-j v}(3+|j|)^{1 / \alpha+\eta} \sum_{k \in D_{j}^{2}}(1+|k|)^{-(2-1 / \alpha-\eta)} . \\
& \leq C \sum_{j=j_{0}+1}^{+\infty} 2^{-j(1+v-1 / \alpha-\eta)}(3+|j|)^{1 / \alpha+\eta} \leq C 2^{-\left(j_{0}+1\right)(1+v-1 / \alpha-\eta)}\left(2+j_{0}\right)^{1 / \alpha+\eta} \\
& \leq C 2^{-\left(j_{0}+1\right)(v-1 / \alpha)} \sup _{n \in \mathbb{N}}\left\{2^{-n(1-\eta)}(1+n)^{1 / \alpha+\eta}\right\} \leq C_{4}\left|u_{1}-u_{2}\right|^{v-1 / \alpha} .
\end{aligned}
$$

On the other hand, using Proposition 2.5, one has

$$
\begin{aligned}
& \sum_{j=j_{0}+1}^{+\infty} 2^{-j v} \sum_{k \in D_{j}^{1}}\left|\epsilon_{j, k}\right|\left(\left(3+\left|2^{j} u_{1}-k\right|\right)^{-2}+\left(3+\left|2^{j} u_{2}-k\right|\right)^{-2}\right) \\
& \leq C^{\prime} \sum_{j=j_{0}+1}^{+\infty} 2^{-j(v-1 / \alpha)} \sum_{k \in D_{j}^{1}}\left(\left(3+\left|2^{j} u_{1}-k\right|\right)^{-2}+\left(3+\left|2^{j} u_{2}-k\right|\right)^{-2}\right) \\
& \leq C^{\prime}\left(2 \sup _{x \in[0,1]} \sum_{k \in \mathbb{Z}}(3+|x-k|)^{-2}\right) \sum_{j=j_{0}+1}^{+\infty} 2^{-j(v-1 / \alpha)} \\
& \leq C_{5} 2^{-\left(j_{0}+1\right)(v-1 / \alpha)} \leq C_{5}\left|u_{1}-u_{2}\right|^{v-1 / \alpha} .
\end{aligned}
$$

Next combining (40) and (41), one obtains that

$$
B_{j_{0}}\left(u_{1}, u_{2}, v\right) \leq C_{6}\left|u_{1}-u_{2}\right|^{v-1 / \alpha},
$$

where $C_{6}$ is a positive and finite random variable. Finally, Relations (33), (39) and (42) allow us to derive (28).

Proof of Proposition 2.3. Let $\omega \in \Omega_{0}^{*}$ be arbitrary and fixed. In view of Lemma 2.1 and of Remark 2.2 in Section 2, for proving the proposition, it is enough to show 
that, for all fixed integer $r \geq 1$ and for any fixed $(p, q) \in\{1,2,3\} \times \mathbb{Z}^{+}$, the two series of real numbers

$$
\sum_{j=1}^{+\infty} \sum_{k \in \mathbb{Z}} \epsilon_{-j, k}(\omega) 2^{j v}\left(\left(\partial_{v}^{r} \Psi\right)\left(2^{-j} u-k, v\right)-\left(\partial_{v}^{r} \Psi\right)(-k, v)\right)
$$

and

$$
\sum_{j=1}^{+\infty} \sum_{k \in \mathbb{Z}} \epsilon_{-j, k}(\omega) 2^{-j(p-v)}\left(\partial_{u}^{p} \partial_{v}^{q} \Psi\right)\left(2^{-j} u-k, v\right)
$$

are uniformly convergent when $(u, v) \in[-M ; M] \times[a ; b]$, where $M, a, b$ arbitrary and fixed real numbers satisfying $M>0$ and $1 / \alpha<a<b<1$. In fact it is sufficient to show that these two series are normally convergent, in sense of the uniform (semi)-norm on the compact rectangle $[-M ; M] \times[a ; b]$. That is one has, for all fixed integer $r \geq 1$,

$$
\sum_{j=1}^{+\infty} \sum_{k \in \mathbb{Z}}\left|\epsilon_{-j, k}(\omega)\right| \sup _{(u, v) \in[-M ; M] \times[a ; b]}\left\{2^{j v}\left|\left(\partial_{v}^{r} \Psi\right)\left(2^{-j} u-k, v\right)-\left(\partial_{v}^{r} \Psi\right)(-k, v)\right|\right\}<\infty,
$$

and, one has, for any fixed $(p, q) \in\{1,2,3\} \times \mathbb{Z}^{+}$,

$$
\sum_{j=1}^{+\infty} \sum_{k \in \mathbb{Z}}\left|\epsilon_{-j, k}(\omega)\right| \sup _{(u, v) \in[-M ; M] \times[a ; b]}\left\{2^{-j(p-v)}\left|\left(\partial_{u}^{p} \partial_{v}^{q} \Psi\right)\left(2^{-j} u-k, v\right)\right|\right\}<\infty .
$$

Next, observe that a sufficient condition for having (43) is that (44) holds when $(p, q)=(1, r)$. Indeed, it follows from the mean value theorem that

$$
\begin{aligned}
& \sup _{(u, v) \in[-M ; M] \times[a ; b]}\left\{2^{j v}\left|\left(\partial_{v}^{r} \Psi\right)\left(2^{-j} u-k, v\right)-\left(\partial_{v}^{r} \Psi\right)(-k, v)\right|\right\} \\
& \leq M \times \sup _{(u, v) \in[-M ; M] \times[a ; b]}\left\{2^{-j(1-v)}\left|\left(\partial_{u}^{1} \partial_{v}^{r} \Psi\right)\left(2^{-j} u-k, v\right)\right|\right\} .
\end{aligned}
$$

So, from now on, our goal is to derive (44). One denotes by $[\cdot]$ the integer part function and by $\{\cdot\}$ the fractional part function. One assumes that $\eta$ is an arbitrarily small fixed positive real number. By using Relation (11), Relation (10) and the triangle inequality, one has, for all $(u, v) \in[-M ; M] \times[a ; b]$,

$$
\begin{aligned}
& \sum_{j=1}^{+\infty} \sum_{k \in \mathbb{Z}}\left|\epsilon_{-j, k}(\omega)\right| \times 2^{-j(p-v)}\left|\left(\partial_{u}^{p} \partial_{v}^{q} \Psi\right)\left(2^{-j} u-k, v\right)\right| \\
& \leq C(\omega) \sum_{j=1}^{+\infty} 2^{-j(p-v)}(3+j)^{1 / \alpha+\eta} \sum_{k \in \mathbb{Z}} \frac{(3+|k|)^{1 / \alpha+\eta}}{\left(3+\left|2^{-j} u-k\right|\right)^{2}} \\
& =C(\omega) \sum_{j=1}^{+\infty} 2^{-j(p-v)}(3+j)^{1 / \alpha+\eta} \sum_{k \in \mathbb{Z}} \frac{\left(3+\left|k+\left[2^{-j} u\right]\right|\right)^{1 / \alpha+\eta}}{\left(3+\left|2^{-j} u-\left[2^{-j} u\right]-k\right|\right)^{2}} \\
& \leq C(\omega) \sum_{j=1}^{+\infty} 2^{-j(p-b)}(3+j)^{1 / \alpha+\eta} \sum_{k \in \mathbb{Z}} \frac{(4+|k|+M \mid)^{1 / \alpha+\eta}}{\left(3+\left|\left\{2^{-j} u\right\}-k\right|\right)^{2}} .
\end{aligned}
$$

Notice that the last inequality follows from the inequalities $v \leq b$ and $\left|\left[2^{-j} u\right]\right| \leq$ $1+2^{-j}|u| \leq 1+M$. Also notice that $C(\omega)$ is a finite constant not depending on $(u, v)$. Next, let $\kappa$ be the constant defined as:

$$
\kappa:=\sup _{x \in[0 ; 1]}\left\{\sum_{k \in \mathbb{Z}} \frac{(4+|k|+M \mid)^{1 / \alpha+\eta}}{(3+|x-k|)^{2}}\right\} .
$$


From the inequalities $2-1 / \alpha-\eta>1$ and $3+|x-k| \geq 2+|k|$, for all $(k, x) \in \mathbb{Z} \times[0,1]$, one deduces that $\kappa$ is finite. Then, using our previous calculations, one obtains that

$$
\begin{aligned}
& \sum_{j=1}^{+\infty} \sum_{k \in \mathbb{Z}}\left|\epsilon_{-j, k}(\omega)\right| \sup _{(u, v) \in[-M ; M] \times[a ; b]}\left\{2^{-j(p-v)}\left|\left(\partial_{u}^{p} \partial_{v}^{q} \Psi\right)\left(2^{-j} u-k, v\right)\right|\right\} \\
& \leq \kappa C(\omega) \sum_{j=1}^{+\infty} 2^{-j(p-b)}(3+j)^{1 / \alpha+\eta}<\infty
\end{aligned}
$$

where the last inequality results from the inequalities $p \geq 1>b$. This proves that (44) holds.

\section{Acknowledgements}

The authors are very grateful to the editor and to the anonymous associate editor and two referees for their valuable comments and suggestions which have led to great improvements of the article. This work has been partially supported by ANR-11-BS01-0011 (AMATIS), GDR 3475 (Analyse Multifractale), and ANR-11LABX-0007-01 (CEMPI).

\section{References}

Ayache, A. and Hamonier, J. (2014) Linear multifractional stable motion: fine path properties Rev. Mat. Iberoam, 30, 1301-1354.

Ayache, A. and Jaffard, S. and Taqqu, M.S. (2007) Wavelet construction of generalized multifractional processes Rev. Mat. Iberoam, 23, 327-370.

Ayache, A. and Roueff, F. and Xiao, Y. (2009) Linear fractional stable sheets: wavelet expansion and sample path properties Stochastic Process. Appl., 119, $1168-1197$.

Ayache, A. and Shieh, N.R. and Xiao, Y. (2011) Multiparameter multifractional Brownian motion: local nondeterminism and joint continuity of the local times Ann. Inst. Henri Poincaré (B) Probab. Stat., 47, 1029-1054.

Ayache, A. and Taqqu, M.S. (2005) Multifractional processes with random exponent Publ. Mat., 49, 459-486.

Balança, P. (2014) Fine regularity of Lévy processes and linear (multi)fractional stable motion, Electron. J. Probab.,19, 1-37.

Balança, P. (2015) Some sample path properties of multifractional Brownian motion, tochastic Process. Appl.,125, 3823-3850.

Bardet, J.M. and Surgailis, D.(2013) Nonparametric estimation of the local Hurst function of multifractional Gaussian processes Stochastic Process. Appl., 123, 1004-1045.

Benassi, A. and Jaffard, S. and Roux, D. (1997) Elliptic Gaussian random processes Rev. Mat. Iberoam, 13, 19-90. 
Biermé, H. and Lacaux, C. (2013) Linear multifractional multistable motion: LePage series representation and modulus of continuity Annals of the University of Bucharest (mathematical series),LXII, 4, 345-360.

Bianchi, S. (2005) Pathwise identification of the memory function of multifractional Brownian motion with application to finance Int. J. Theor. Appl. Finance, 8, 255-281.

Bianchi, S. and Pantanella, A. and Pianese, A. (2011) Modeling stock prices by multifractional Brownian motion: an improved estimation of the pointwise regularity Quant. Finance, 13, 1317-1330.

Bianchi, S. and Pianese, A. (2008) Multifractional properties of stock indices decomposed by filtering their pointwise Hölder regularity Int. J. Theor. Appl. Finance, 11, 567-595.

Daubechies, I. (1992) Ten lectures on wavelets, Society for Industrial Mathematics, 61.

Dozzi, M. and Shevchenko, G. (2011) Real harmonizable multifractional stable process and its local properties Stochastic Process. Appl., 121, 1509-1523.

Falconer, K.J. (2002) Tangent fields and the local structure of random fields $J$. Theoret. Probab., 15, 731-750.

Falconer, K.J. (2003) The local structure of random processes J. London Math. Soc. (2), 67, 657-672.

Falconer, K.J. and Le Guével, R. and Lévy Véhel, J. (2009) Localizable moving average symmetric stable and multistable processes Stoch. Models, 25, 648-672.

Falconer, K.J. and Lévy Véhel, J. (2009) Multifractional, multistable, and other processes with prescribed local form J. Theoret. Probab., 22, 375-401.

Hashorva, E. and Lifshits, M. and Seleznjev, O. (2015) Approximation of a random process with variable smoothness Mathematical Statistics and Limit Theorems, Springer, 189-208.

Kôno, N. and Maejima, M. (1991) Hölder continuity of sample paths of some self-similar stable processes Tokyo J. Math., 14, 93-100.

Lacaux, C. (2004) Real harmonizable multifractional Lévy motions Ann. Inst. H. Poincaré (B) Probab. Stat., 40, 259-277.

Leonenko, N. and Ruiz-Medina, M.D. and Taqqu, M.S. (2011) Fractional elliptic, hyperbolic and parabolic random fields Electron. J. Probab., 16, 1134-1172.

Lopes, R. and Ayache, A. and Makni, N. and Puech, P. and Villers, A. and Mordon, S. and Betrouni, N. (2011) Prostate cancer characterization on images using fractal features Medical Physics, 38, 83-95.

Maejima, M. (1983) A self-similar process with nowhere bounded sample paths, Probability Theory and Related Fields, 65, 1, 115-119.

Meerschaert, M. and Wu, D. and Xiao, Y. (2008) Local times of multifractional Brownian sheets Bernoulli, 14, 865-898.

Meyer, Y. (1990) Ondelettes et Opérateurs , 1, Hermann, Paris. 
Meyer, Y. (1992) Wavelets and operators, 2, Cambridge Univ Press.

Peltier, R.F. and Lévy Véhel, J. (1995) Multifractional Brownian motion: definition and preliminary results Rapport de recherche de l'INRIA, 2645.

Samorodnitsky, G. and Taqqu, M. S. (1994) Stable non-Gaussian Random Variables, Chapman and Hall, London.

Stoev, S. and Taqqu, M. S. (2004) Stochastic properties of the linear multifractional stable motion Adv. in Appl. Probab. 36, 1085-1115.

Stoev, S. and Taqqu, M. S. (2005) Path properties of the linear multifractional stable motion Fractals 13, 157-178.

Stoev, S. and Taqqu, M. S. (2006) How rich is the class of multifractional Brownian motions? Stochastic Process. Appl. 116, 200-221.

Surgailis, D. (2008) Nonhomogeneous fractional integration and multifractional processes Stochastic Process. Appl. 118, 171-198.

Takashima, K. (1989) Sample path properties of ergodic self-similar processes Osaka J. Math. 26, 159-189. 